\title{
Problemas de sueño y síntomas de trastornos de la conducta alimentaria (TCA) en mujeres jóvenes
}

\author{
Klaus Seigel, MD \\ Jan-Erik Broman, PhD \\ Jerker Hetta, MD PhD \\ Department of Neuroscience (Psychiatry) \\ Uppsala University, University Hospital of \\ Uppsala, SE-751 85 Uppsala \\ SWEDEN
}

\begin{abstract}
RESUMEN - Objetivo: Evaluar la prevalencia de algunos problemas de sueño y de síntomas de trastornos de la conducta alimentaria (TCA), así como las posibles asociaciones entre ellos, en una población de mujeres jóvenes.

Método: Cuestionario autoadministrado en una muestra aleatoria de 726 mujeres, de entre 18 y 23 años. Se compararon dos grupos, con y sin determinados tipos de problemas de sueño, en relación a síntomas de TCA.

Resultados: Un 10,7\% de la muestra refirieron problemas subjetivos graves de sueño, un $16,2 \%$ indicios de atracón, y un 6,7\% necesidad impulsiva de vomitar tras las comidas. Los problemas de sueño específicamente asociados con síntomas de TCA fueron las dificultades para mantener el sueño y la sensación de no haber tenido un sueño reparador. En contra de lo esperado, no se encontró asociación entre el despertar precoz y los síntomas TCA, ni tampoco con un bajo Índice de Masa Corporal.

Conclusiones: Los síntomas de TCA entre mujeres jóvenes parecen estar asociados a la dificultad para mantener el sueño y a la sensación de no haber tenido un sueño reparador.
\end{abstract}

\section{Introducción}

Los problemas de sueño son frecuentes entre los jóvenes, especialmente entre las mujeres (Husby y Lingjaerde 1990, Coren 1994, Jansson et al. 1995, Lindberg et al. 1997, Manni et al. 1997). En el estudio de Lindberg et al. (1997) se asocia el género femenino con problemas de sueño, incluso tras ajustar por status psicológico.

También son frecuentes en mujeres jóvenes síntomas de trastornos de la conducta alimentaria (TCA) tales como: preocupación por el peso, restricción en la ingesta, dietas frecuentes, atracones y conductas purgativas (Shefer 1987, Moore 1988, Cas- 
per y Offer 1990, Cooper y Fairburn 1993, Story et al. 1994).

En relación con las asociaciones entre problemas de sueño y TCA, los estudios de laboratorio (Walsh et al. 1985, Dippel et al. 1987, Hudson et al. 1987, Levy, Dixon y Schmidt 1987, Levy, Dixon y Schmidt 1988, Delvenne et al. 1992) documentaron que las pacientes no deprimidas con anorexia o bulimia nerviosa tienen registros de sueño muy similares a sujetos normales. Sin embargo, Walsh et al. (1985), así como Delvene et al. (1992), documentaron en pacientes anoréxicas una menor eficiencia del sueño, algunos problemas de insomnio intermedio y una menor duración del sueño. En los años sesenta y setenta (Crisp 1967, Crisp y Stonehill 1973) se encontró una asociación entre pérdida de peso y despertar precoz, en sujetos no deprimidos con anorexia nerviosa y otros trastornos, y dicho trastorno de sueño es un ítem del "Eating Attitudes Test” (EAT) (Garner y Garfinkel, 1979).

A pesar de su prevalencia, no hay estudios sobre la co-morbilidad y las asociaciones entre los problemas de sueño y los síntomas de TCA en población general de mujeres jóvenes. En el contexto de un proyecto más general nos planteamos llevar a cabo un estudio de este tipo.

\section{Método}

\section{Muestra}

Se seleccionó una muestra simple aleatoria a partir del censo sueco de 1.000 mujeres entre 18 y 23 años en el condado de Uppsala, Suecia. Se obtuvo respuesta de 726 . Puesto que 23 sujetos no recibieron el cuestionario debido a un cambio o a un error en su dirección, la tasa de respuesta fue de 726/977 = 0,743 . La edad media de las entrevistadas fue de 20,2 \pm 1,8 años. Casi cuatro de cada cinco entrevistadas eran estudiantes. (No encontramos asociaciones estadísticamente significativas entre variables sociales y síntomas de problemas de sueño o de TCA). Se solicitó el consentimiento informado a las entrevistadas asegurando su participación anónima. El Comité de Ética de la Facultad de Medicina de la Universidad de Uppsala aprobó el diseño del estudio. Las participantes respondieron el cuestionario entre los meses de febrero y abril de 1998.

\section{Cuestionario}

En la Tabla I se enumeran la mayoría de los ítems usados. Se instruyó a las entrevistadas para responder al cuestionario indicando cómo se habían sentido en los tres meses previos a la entrevista.

Mientras que los ítems de sueño fueron tomados de un instrumento de screening (no una escala) previo para problemas de sueño, el Uppsala Sleep Inventory (Inventario de sueño de Uppsala) (Hetta et al. 1985), la mayoría de los ítems referidos a TCA se diseñaron ex-novo. También se tomaron dos ítems del Uppsala Sleep Inventory sobre la autoestimación de la duración normal y necesaria del sueño. Además, el cuestionario incluyó ítems acerca de la edad, altura, peso y situación menstrual ("sangrado regularirregular-no sangrados al menos de tres meses-embarazo") e ítems para valorar aspectos sociales (ocupación principal, nivel de educación y situación de convivencia).

\section{Análisis factorial y puntuaciones compuestas}

Para documentar la validez de constructo del cuestionario, y para reducir el número de variables que exploran otros síntomas 


\section{Tabla I}

Listado de ítems usados en el análisis factorial*

Formulación de las preguntas

Posibles respuestas

IMAGEN CORPORAL E INGESTA*

¿Cuántas veces ${ }^{\dagger}$ has intentado bajar de peso?

¿Con qué frecuencia...

Nunca / $1-5$ veces / 6-10 veces / >10 veces

...temes ganar peso?

... sientes que tienes sobrepeso?

...te sientes insatisfecha con tu cuerpo?

...ingieres grandes cantidades de comida con pérdida de autocontrol?

...sientes impulsos de vomitar después de las comidas?

Nunca / raramente / a veces / frecuentemente / muy frecuentemente

$$
\begin{aligned}
& -",- \\
& -"- \\
& -"- \\
& -"- \\
& -"-
\end{aligned}
$$

\section{INSOMNIO*}

¿Cómo de graves son tus problemas de ...

...dificultad para conciliar el sueño?

...despertar frecuentemente por la noche?

...despertar demasiado pronto por la mañana?

...No haber tenido un sueño reparador?

Nada / poco / moderado / grave / muy grave

$$
\begin{aligned}
& -"{ }_{-} \\
& -"-- \\
& -"- \\
& -"-
\end{aligned}
$$

\section{ACTIVIDAD Y PERFECCIÓN*}

¿Con qué frecuencia...

Nunca / raramente / a veces / frecuentemente /

...piensas o piensan otros que tu nivel general de actividad es demasiado alto?

...sientes que no has descansado?

...quieres hacer las cosas de manera perfecta o si no es mejor no hacerlas?

\section{DEPRESIÓN*}

...tienes pensamientos de suicidio?

...has sentido pánico?

...te sientes como si valieras menos que las demás?

...te sientes triste?

...tienes pensamientos obsesivos? muy frecuentemente

$$
\begin{aligned}
& -"- \\
& -"- \\
& -"-
\end{aligned}
$$
ANSIEDAD*
...te sientes nerviosa?
....sientes estrés?
...estás preocupada?
Nunca / raramente / a veces / frecuentemente / muy frecuentemente

\footnotetext{
* Los encabezados, en mayúsculas, indican ítems que se han agrupado en el análisis factorial y se han utilizado para ítems compuestos.

$\dagger$ Intentos de duración superior a una semana.

\#“Pensamientos desagradables que son difíciles para deshacerse de ellos” (formulación adicional proporcionada a las entrevistadas).
}

Nunca / raramente / a veces / frecuentemente / muy frecuentemente

$$
\begin{aligned}
& -"{ }_{-} \\
& -"-- \\
& -"- \\
& -"- \\
& -"-
\end{aligned}
$$

diferentes de los del sueño y de TCA, se calcularon puntaciones compuestas de aquellos ítems que en el mismo factor obtuvieron un peso con coeficientes $>0,5$ (matriz de solu- ción oblicua). Estos grupos de ítems están señalados en la Tabla I.

Se utilizó un análisis factorial de componentes principales como el método de extrac- 
ción de factores y transformaciones ortotran/varimax. El análisis dio como resultado cinco factores con eigenvalues $>1,0$. (Otros datos básicos: $\mathrm{N}=707$, número de ítems 21 , suma de la proporción de varianza 0,62 , grados de libertad 203, p > 0,0001). Todos los ítems excepto dos se agruparon en un factor, y no hubo ítems agrupados en más de un factor.

\section{Categorías}

Para presentar los resultados de forma fácilmente comprensible en su totalidad y para calcular las Odds Ratio (OR), se construyeron las "categorías problema" usando el punto de corte "grave" para los ítems de sueño, y "bastante a menudo" para los ítems de TCA. Para el ítem referido a "tentativas frecuentes de reducir peso" el punto de corte fue el de "6-10 intentos". Para el IMC (índice de masa corporal) $\left(\mathrm{kg} / \mathrm{m}^{2}\right)$ se consideró normal el intervalo 19,1-24,0 (Hammer et al. 1991). Se consideró el IMC < 17,5 , que se corresponde con "bajo peso grave", con más del $15 \%$ de reducción del IMC normal, según el criterio DSM IV para anorexia nerviosa (American Psychiatric Association 1994).

\section{Métodos estadísticos}

El programa estadístico empleado fue el Stat View 5.0 para Windows. Se documentan las medias \pm una desviación estándar. El nivel de significación usado fue para $\mathrm{p}<0,5$. Para obtener los coeficientes de correlación entre puntaciones se usó el método de Pearson. Para calcular los valores OR, incluyendo sus intervalos de confianza al 95\%, se usaron modelos de regresión logística simple y múltiple, que incluyeron el test de Walds y el modelo logístico completo de "ajuste perfecto" de significación.

\section{Resultados}

\section{Correlación de las puntuaciones compuestas}

Como se muestra en la Tabla II, los coeficientes entre las puntuaciones de insomnio y de TCA fueron los más bajos en la matriz de correlación. Tanto las puntuaciones de insomnio como las de imagen corporal y TCA obtuvieron coeficientes de correlación de alrededor de 0,40 para las puntuaciones de depresión.

Tabla II

Coeficientes de correlación entre puntuaciones de los ítems

\begin{tabular}{llccc} 
& Insomnio & $\begin{array}{c}\text { Imagen corporal \& } \\
\text { TCA }\end{array}$ & Depresión & $\begin{array}{c}\text { Actividad \& } \\
\text { perfeccionismo }\end{array}$ \\
\hline Imagen corporal y TCA & 0,22 & & & \\
Depresión & 0,39 & 0,43 & & \\
Actividad y perfeccionismo & 0,23 & 0,28 & 0,34 & 0,37 \\
Estrés & 0,35 & 0,30 & 0,57 & \\
\hline
\end{tabular}

$\mathrm{N}=709$

IC $95 \% \pm 0,06$

Todos $\mathrm{p}<0,0001$ 


\section{Frecuencia de problemas de sueño}

El problema de sueño más frecuente fue no haber tenido un sueño reparador, referido por el $17,3 \%$, seguido por dificultad para conciliar el sueño $6,5 \%$, dificultad para mantener el sueño (despertarse frecuentemente por la noche) $4,7 \%$, y despertar pre$\operatorname{coz} 3,3 \%$. Sin diferenciar entre los tres últimos tipos, el 10,7\% aquejó tales problemas o "insomnio" de forma grave o muy grave.

Los valores medios para duración del sueño normal y necesitada fueron de 7,43 horas \pm 1,05 y 8,43 horas $\pm 1,13$, respectivamente. El cociente de éstos, con criterios Sleep Sufficiency Index (Índice de Sueño Suficiente), fue de 0,88 (Broman, Lundh y Hetta 1996).

\section{Frecuencia de TCA}

El valor medio de IMC fue de 21,7 $\pm 3,0$. De todas las entrevistadas, un $16,4 \%$ podría considerarse con sobrepeso. Un 13,2\% obtuvieron valores IMC inferiores al normal, mientras que un $1,8 \%$ tuvo un IMC por debajo de 17,5.

Un $15,3 \%$ de la muestra presentó más de cinco intentos previos de reducir el peso. Indicios de atracón e impulsos de vomitar tras la ingesta fueron referidos por un $16,2 \%$ y un $6,7 \%$, respectivamente.

Problemas de imagen corporal como temor a ganar peso afectaron a un $41,6 \%$, sentirse con sobrepeso a un $33,1 \%$ e insatisfacción con el cuerpo a un $28,7 \%$ de la muestra.

\section{Asociaciones entre problemas de sueño y TCA}

Los problemas de imagen corporal, intentos frecuentes por reducir peso, indicios de atracón e impulsos para vomitar después de las comidas se asociaron todos significativamente con las dificultades para mantener el sueño y con la sensación de no haber tenido un sueño reparador (Tabla III). En la muestra total, un 4,6\% refirió una sintomatología combinada de indicios de atracón e impulsos de vomitar tras la ingesta. Esta combinación de síntomas se asoció con dificultad para mantener el sueño y, en menor grado, con despertar precoz, OR 6,9 (2,7 - 17,3) $\mathrm{p}<0,0001$ y OR 4,6 $(1,5-14,4) \mathrm{p}<0,01$, respectivamente.

Las desviaciones del IMC, incluyendo bajo peso grave o problemas menstruales, no produjeron resultados significativos con las variables de sueño. No hubo asociaciones significativas entre TCA y duración del sueño.

\section{Discusión}

Los resultados indican que los problemas subjetivos graves de dificultad para mantener el sueño y sensación de no haber tenido un sueño reparador se asocian con problemas de imagen corporal, intentos recurrentes de perder peso y con tendencias bulímicas entre las mujeres jóvenes.

Nuestros resultados acerca de la frecuencia de problemas de sueño y de TCA están en la línea de investigaciones anteriores. Janson et al. (1995) y Lindberg et al. (1997) han documentado respectivamente dificultad para conciliar el sueño en un 6-9\% y un $4,8 \%$ de sus entrevistadas, de entre 20 y 45 años. Nuestro resultado fue de un 6,5\%. De forma similar, se encontró despertar precoz en el 5-6\% de su muestra mientras que nosotros obtuvimos un $3,3 \%$.

La gente joven también tiende a dormir menos de lo que necesita; un estudio de 


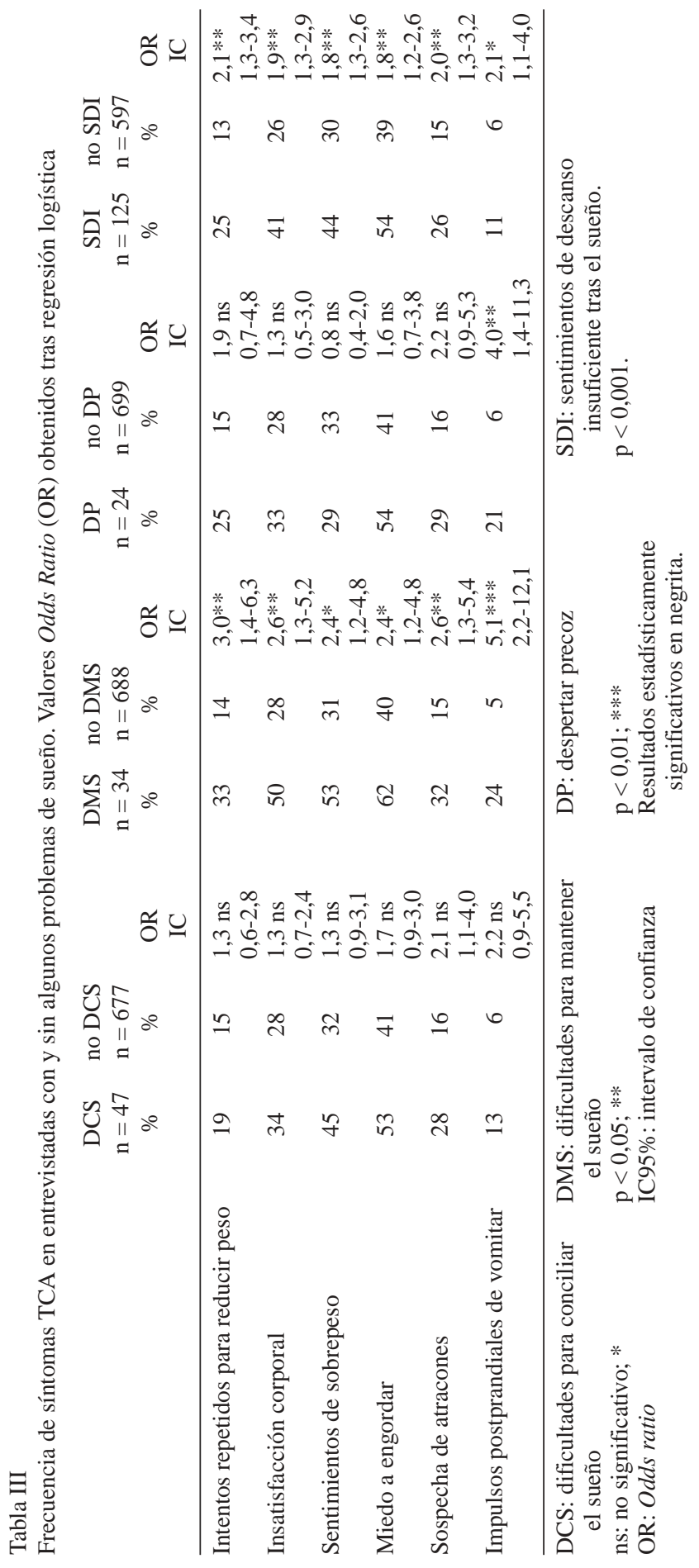


Strauch y Meier (1988) mostró una discrepancia entre la duración del sueño normal y deseada de 1,7 horas en los días entre semana y un deseo de dormir más entre un 54 y un $75 \%$ de los sujetos. En nuestro estudio, el $72 \%$ refirieron un déficit subjetivo de tiempo de sueño.

Las frecuencias de TCA en la muestra pueden parecer irrealmente altas, lo que va en contra de la credibilidad de los resultados; sin embargo, los "síndromes TCA parciales" son mucho más comunes que la anorexia o bulimia nerviosas y parecen estar relacionados con estos trastornos clínicos siguiendo un modelo de continuum (Dancyger y Garfinkel 1995). Para ejemplificar esto, un $16 \%$ y un $7 \%$ de nuestra muestra se vio afectado por indicios de atracón e impulso de vomitar después de las comidas, y podrían ser comparados con un $22 \%$ para atracón y un $6 \%$ para vómito autoinducido para controlar el peso encontrados por Shefer entre estudiantes femeninas en Sudáfrica (1987). También podrían compararse con un $21 \%$ para atracón y un $3 \%$ para vómito documentado por Cooper y Fairburn entre mujeres jóvenes que acudían a un consultorio de planificación familiar en el Reino Unido (Cooper y Fairburn 1993).

En relación con los problemas de imagen corporal, estudios anteriores han mostrado que son más frecuentes en la población femenina, y las estimaciones de prevalencia se encuentran en un rango entre 40-65\% dependiendo de la población y los métodos (Moore 1988, Casper y Offer 1990, Store et al. 1994). Nuestros resultados indican que aproximadamente un tercio de la población femenina teme a menudo ganar peso, están insatisfechas con su cuerpo o se sienten con sobrepeso. Casi dos tercios de las entrevistadas que refirieron sentirse con sobrepeso en realidad tenían un IMC normal o bajo.

Respecto a la coexistencia de problemas de sueño y TCA, no son posibles las compa- raciones con resultados anteriores, ya que no se han encontrado estudios poblacionales en este tema. Aunque se han obtenido asociaciones estadísticamente significativas entre algunos problemas de sueño y algunos síntomas de TCA e imagen corporal, sus valores son modestos.

En relación a que la dificultad para mantener el sueño sea el insomnio más típico de los individuos con TCA, un estudio de laboratorio de Maes et al. (1993) es relevante para la discusión. El estudio mostró que la dificultad para mantener el sueño en sujetos deprimidos se asociaba a signos indirectos de aumento del recambio de catecolaminas, ansiedad somática e hipocondriasis, "un estado de hiperalerta". Nuestra hipótesis es que tales datos de laboratorio podrían conducir a resultados similares para sujetos con preocupación por su imagen corporal y tendencias bulímicas, aquejados de dificultad para mantener el sueño.

En relación con la asociación entre pérdida de peso y despertar precoz en sujetos con anorexia nerviosa y otros, documentada por Crisp (1967) y por Crisp y Stonehill (1973), encontramos una asociación significativa entre despertar precoz e impulsos de vomitar después de las comidas, pero no para con otras variables de TCA, incluyendo bajo peso grave. Además, cuando consideramos los intentos actuales de reducir peso e IMC juntas como variables independientes en modelos de regresión logística múltiple con variables de sueño como variables dependientes, sólo se observó una reducción ligera, de aproximadamente un $10-15 \%$ de la OR. Este efecto no fue mayor con "despertar precoz" que con otras variables de sueño. Sin embargo, los ítems que aportan información sobre la rapidez de la pérdida de peso podrían habernos dado mejores herramientas para poner en duda las conclusiones de estos investigadores. 
No podemos llegar a ninguna conclusión en relación a las relaciones causales entre los problemas de sueño y TCA. Aunque probablemente estos dos tipos de síntomas se influyen entre sí, se necesitan futuras investigaciones para describir cómo. Además, como indican los coeficientes de correlación, mientras que los problemas de sueño y los TCA parecen estar asociados modestamente entre sí, sus respectivas asociaciones con síntomas de depresión, ansiedad y estrés parecen ser notablemente más fuertes.

Por lo que concierne a las limitaciones, un problema metodológico es si usar ítems que preguntan por la frecuencia de determinados síntomas, o por la gravedad subjetiva de los mismos. Puesto que los problemas subjetivos son precisamente los que llevan al paciente al médico, hemos considerado tales ítems de interés clínico.

Se decidió usar las variables de sueño categorizadas y las variables de TCA para proporcionar una presentación comprensible de la frecuencia y coexistencia de los síntomas. Encontrar los puntos de corte apropiados para agrupar es un problema cuando se hacen categorías, pero puesto que las correlaciones entre los datos brutos fueron lineales, los cálculos alternativos utilizando de alguna manera diferentes puntos de corte no cambiaron el patrón global de los resultados.

En cuanto a la representatividad de nuestra muestra aleatoria de mujeres jóvenes, la frecuencia de sujetos que estudiaban en niveles superiores fue alta. Esto es de esperar ya que Uppsala es una ciudad de educación superior. Sin embargo, no encontramos ninguna tendencia para los problemas de sueño o de TCA relacionadas con el nivel educacional en nuestro estudio.

En conclusión, los intentos recurrentes para perder peso, los problemas de imagen corporal y las tendencias bulímicas parecen estar asociadas con problemas graves de dificultad para mantener el sueño y la sensación de no haber tenido un sueño reparador.

\section{Bibliografía}

AMERICAN PSYCHIATRIC ASSOCIATION. Diagnostic and statistical manual of mental disorders, $4^{\text {th }}$ edn. American Psychiatric Association. Washington DC 1994.

BROMAN, J.E., LUNDH, L.G., HETTA, J. Insufficient sleep in the general population. Clinical Neurophysiology 26: 30-39, 1996.

CASPER, R.C., OFFER, D. Weight and dieting concerns in adolescents, fashion or symptom? Paediatrics 86 : 384-390, 1990.

COOPER, P.J., FAIRBURN, C.G. Binge-eating and self-induced vomiting in the community. A preliminary study. British Journal of Psychiatry 142: 139-144, 1993.

COREN, S. The prevalence of self-reported sleep disturbances in young adults. International Journal of Neuroscience 79: 67-73, 1994.

CRISP, A.H. The possible significance of some behavioural correlates of weight and carbohydrate intake. Journal of Psychosomatic Research 11: 117- 131, 1967.

CRISP, A.H., STONEHILL, E. Aspects of the relationship between sleep and nutrition: A study of 375 psychiatric out-patients. British Journal of Psychiatry 122: 379394, 1973.

DANCYGER, I.F., GARFINKEL, P.E. The relationship of partial syndrome eating disorders to anorexia nervosa and bulimia nervosa. Psychological Medicine 25: 10191025, 1995.

DELVENNE, V., KERKHOFS, M., APPELBOOMFONDU, J., LUCAS, F., MENDLEWICZ, J. Sleep polygraphic variables in anorexia nervosa and depression: a comparative study in adolescents. Journal of Affective Disorders 25: 167- 172, 1992.

DIPPEL, B., LAUER, C., RIEMANN, D., MAJER TRENDEL, K., KRIEG, J.C., BERGER, M. Sleep and dreams in eating disorders. Psychotherapy and Psychosomatics 48: 165-169, 1987.

GARNER, D.M., GARFINKEL, P.E. The Eating Attitudes Test: An index of the symptoms of anorexia nervosa. Psychological Medicine 9: 273-279, 1979. 
HAMMER, L.D., KRAEMER, H.C., WILSON, D.M., RITTER, P.L., DORNBUSCH, S.M. Standardized percentile curves of body-mass index for children and adolescents. American Journal of Disabled Children 145: 259263, 1991.

HETTA, J., ALMQVIST, M., ÅGREN, H., HAMBERT, G., LILJENBERG, G.B., ROOS, B.E. Prevalence of sleep disturbances and related symptoms in a middle-aged Swedish population. In: Sleep '84, 373-376. Gustaf Fischer Verlag. 1985.

HUDSON, J.I., POPE, H.G. JR., JONAS, J.M., STAKES, J.W, GROCHOCINSKI, V., LIPINSKI, J.F., KUPFER, D. J. Sleep EEG in bulimia. Biological Psychiatry 22: 820-828, 1987.

HUSBY, R., LINGJAERDE, O. Prevalence of reported sleeplessness in northern Norway in relation to sex, age and season. Acta Psychiatrica Scandinavica 81: 542-547, 1990.

JANSON, C., GISLASON, T., DE BACKER, W., PLASCHKE, P., BJORNSSON, E., HETTA, J., KRISTBJARNASON, H., VERMEIRE, P., BOMA, N.G. Prevalence of sleep disturbances among young adults in three European countries. Sleep 18: 589-597, 1995.

LEVY, A.B., DIXON, K.N., SCHMIDT, H. REM and delta sleep in anorexia nervosa and bulimia. Psychiatry Research 20: 189-197, 1987.

LEVY, A.B., DIXON, K.N., SCHMIDT, H. Sleep architecture in anorexia nervosa and bulimia. Biological Psychiatry 23: 99-101, 1988.

LINDBERG, E., JANSON, C., GISLASON, T., BJÖRNSSON, E., HETTA, J., BOMAN, G. Sleep disturbances in a young adult population: can gender differences be explained by differences in psychological status? Sleep 20: 381-387, 1997.

MAES, M., MELTZER, H.Y., SUY, E., MINNER, B., CALABRESE, J., COSYNS, P. Sleep disorders and anxiety as symptom profiles of sympathoadrenal system hyperactivity in major depression. Journal of Affective Disorders 27: 197-207, 1993.

MANNI, R., RATTI, M.T., MARCHIONI, G., CASTELNOVO, G., MURELLI, R., SARTORI, I., GALIMBERTI, C.A., TARTARA, A. Poor sleep in adolescents: a study of 869 17-year-old Italian secondary school students. Journal of Sleep Research 6: 44-49, 1997.

MOORE, D.C. Body image and eating behaviour in adolescent girls. American Journal of Disabled Children 142: 1114-1118, 1988.

SHEFER, T. Abnormal eating attitudes and behaviours among women students. South African Medical Journal 72: 419-421, 1987.

STORY, M., HAUCK, F.R., BROUSSARD, B.A., WHITE, L.L., RESNICK, M.D., BLUM, R.W. Weight perceptions and weight control practices in American Indian and Alaska Native adolescents. A national survey. Archives of Pediatric and Adolescent Medicine 148: 567-571, 1994.

STRAUCH, I., MEIER, B. Sleep need in adolescents: a longitudinal approach. Sleep 11: 378-386, 1988.

WALSH, B.T., GOETZ, R., ROOSE, S.P., FINGEROTH, S., GLASSMAN, A.H. EEG-monitored sleep in anorexia nervosa and bulimia. Biological Psychiatry 20: 947-956, 1985.

Dirección para correspondencia:

Klaus Seigel MD

Department of Neuroscience (Psychiatry) Uppsala University University Hospital of Uppsala

SE-751 85 Uppsala

SWEDEN

E-mail: klaus.seigel@uaspsyk.uu.se 